\title{
Facilitadores y barreras del proceso de inclusión en educación superior: la percepción de los tutores del programa Piane-UC*
}

\author{
Facilitators and barriers of the inclusion process in higher education: \\ the perspective of tutors of Piane-UC program
}

\section{Eugenia Victoriano Villouta}

Asesora Técnica Fundación Mis Talentos

Telf.: (56) 982185719. Correo electrónico: evictoriano@uc.cl

\begin{abstract}
RESUMEN
La educación superior representa mayores posibilidades de participación en la sociedad a personas con discapacidad. En este contexto, para favorecer la inclusión de alumnos con discapacidad motora y sensorial, la Pontificia Universidad Católica de Chile tiene el programa PIANE-UC. El objetivo del presente estudio es identificar y comprender los facilitadores y las barreras en la inclusión de alumnos con discapacidad en el contexto de la educación superior, desde la perspectiva de los alumnos tutores que brindan apoyo en este programa. Para ello, mediante un enfoque cualitativo en la modalidad de estudio de caso, se analizaron nueve entrevistas utilizando la metodología de Teoría Fundamentada. Este análisis reveló que un aspecto importante a considerar es la conceptualización que se tenga de la discapacidad, pues en función de ello se generarán prácticas más o menos inclusivas.
\end{abstract}

Palabras clave: modelo social, tutoría de pares, discapacidad.

\section{ABSTRACT}

Higher education represents greater opportunities for participation in society for people with disabilities. In this context, in order to promote the inclusion of pupils with sensory and motor disabilities Pontificia Universidad Católica de Chile develops the PIANE -UC program. The aim of this study is to identify and understand the facilitators and barriers of the inclusion of students with disabilities in the context of higher education from the perspective of the tutors that support students in this program. Through a qualitative approach in the form of case study, nine interviews were analyzed using Grounded Theory methodology. This analysis revealed that an important aspect to consider is the conceptualization of disability, as bases upon this, the inclusive practices generated will be more or less appropriate.

Key words: social model, peers tutors, disability.

Investigación asociada al Proyecto "Sistematización de la experiencia de trabajo colaborativo entre el Piane-UC y las unidades académicas, para la implementación de adecuaciones curriculares para estudiantes con discapacidad en la UC" de las investigadoras María Rosa Lissi y María Soledad Zuzulich, del PIANE-UC, financiado por la Vicerrectoría Académica y la Pastoral de la Pontificia Universidad Católica de Chile. 


\section{ANTECEDENTES}

Uno de los derechos humanos fundamentales es el derecho a una educación de calidad, ya que permite la supervivencia humana y promueve el acceso a otros derechos sociales (Acedo, 2008 Cit. en García, 2013), por ello, distintos países en el mundo están regulando la no discriminación de las personas en el ámbito educativo, para satisfacer el creciente interés de alumnos con discapacidad por recibir educación universitaria (Konur, 2006). De hecho Abad, Álvarez y Castro de Paz (2008) indican que dentro de las políticas sociales de la Organización de Naciones Unidas (ONU) y en la Unión Europea se hace especialmente relevante el Principio de Igualdad de Oportunidades, el que defiende que todas las personas, independiente de sus diferencias, deben tener acceso a todos los recursos sociales que les garanticen una igualdad de trato y una vida libre de discriminación. Centrándose específicamente en la discapacidad, desde las Naciones Unidas se ha defendido su derecho a la no-discriminación y la igualdad de trato en diversas normativas, entre ellas el Programa de Acción Mundial para las Personas con Discapacidad (1983), las Normas Uniformes sobre la Igualdad de Oportunidades de las Personas con Discapacidad (1993) y la Convención sobre los Derechos de las Personas con Discapacidad (2008). Consistente con esto, Andrade et al. (2011 Cit. en Hockings, Brett \& Terentjevs, 2012) indican que el acceso a la educación debe ser para todos los ciudadanos, y no solo debe ponerse el foco en el acceso, sino que también en las innovaciones que se hagan en favor de estos alumnos, por ejemplo en el acceso a la información.

En el caso de Chile, en febrero de 2010 entró en vigencia la Ley № 20.422 (Ministerio de Planificación, 2010) que garantiza la plena inclusión educacional y social para personas con discapacidad, asegurando el cumplimiento de sus derechos y eliminando cualquier forma de discriminación justificada en esta condición. Entre los artículos de dicha ley, se menciona que las instituciones de educación superior deberán ir, progresivamente, adoptando medidas y mecanismos que faciliten el acceso de las personas con discapacidad, así como también deberán adaptar los materiales de estudio y medios de enseñanza para que dichas personas puedan cursar las diferentes carreras, a fin de que puedan acceder, permanecer y progresar en el sistema de enseñanza superior (García, 2013).

Sin embargo, según el Estudio sobre Inclusión de las Personas con Discapacidad en la Educación Superior en Chile, realizado por el Ministerio de Educación (2011 Cit. en García, 2013) durante el año 2011, se reveló que de 174 instituciones de educación superior que fueron encuestadas acerca de si poseían sistemas adecuados para recibir alumnos en situación de discapacidad, solo 75 instituciones lo contestaron afirmativamente. Estos resultados además arrojaron que si bien estas instituciones cuentan con algún apoyo a los estudiantes con discapacidad, sus falencias se relacionan con una falta de apoyo especial para que los estudiantes cursen las asignaturas, falta de investigación sobre la situación de los alumnos y la necesidad de contar con más sistemas de financiamiento para estos fines.

Lo anterior es reafirmado por Espinoza y González (2008), quienes exponen que pese a que es innegable que este país cuenta con un sistema de educación superior masivo y diversificado que paulatinamente ha ido incrementando su cobertura y oportunidades de acceso en distintos niveles socioeconómicos, aún existen otras formas de discriminación que condicionan el acceso al sistema terciario. Entre estas, se encuentran aquellas relacionadas a la infraestructura o a la falta de mecanismos específicos para el ingreso de estudiantes 
con discapacidad (Instituto Internacional de la UNESCO para la Educación Superior en América Latina y el Caribe [IESALC], 2005). En tanto, Lissi y otros (2009) indican que solo el $6,6 \%$ de los jóvenes con algún tipo de discapacidad accede a la universidad, y cuando lo hacen se encuentran con aspectos específicos que dificultan su inclusión como infraestructura, barreras pedagógicas, comunicacionales y actitudinales.

\section{EL PROBLEMA}

Históricamente, la cuestión de la diferencia y la diversidad, sea cual fuera su origen (de género, cultural, racial, socioeconómica, de orientación sexual o discapacidad/necesidades educativas especiales) ha suscitado innumerables discusiones, una de ellas referida al derecho a la educación Moreno (2006).

En esta línea cabe reiterar que la educación es uno de los derechos humanos fundamentales, así lo rectifica Moreno (2006) planteando que "el derecho de todos de tener acceso a la educación en condiciones de igualdad, es un derecho humano sobre la base del reconocimiento de la diversidad humana" (Moreno, 2006, p. 145).

En este sentido, pese a que a nivel mundial y nacional se han estado haciendo valiosos esfuerzos por promover la inclusión de la diversidad, aún queda pendiente la educación superior, nivel que, según Moreno (2006) ha mostrado ser el más excluyente de todos en el acceso, permanencia y conclusión de estudios. Así lo señalan Dutta, Kundu y Schiro-Geist (2009), indicando que muchos de los alumnos con Necesidades Educativas Especiales (NEE) concluyen sus estudios secundarios y no ingresan a la educación superior debido a la incertidumbre que sienten ante esta transición, por ello estos autores señalan que es fundamental que las universidades establezcan políticas que den lineamientos sobre esto.

Si se considera lo señalado por las Naciones Unidas (2006) respecto a que la discapacidad resulta de la interacción entre las personas con deficiencias y las barreras debidas a la actitud y al entorno que evitan su participación plena y efectiva en la sociedad, en igualdad de condiciones con las demás, es decir, si se conceptualiza como un cruce entre variables que operan en la interacción del individuo con limitaciones y su ambiente físico, social y cultural (Lou, 2011), se hace necesario analizar la relación entre este sujeto con limitaciones y el ambiente en el cual vive, para promover su autonomía, integración y otras capacidades; analizar sus conductas funcionales a fin de identificar los apoyos requeridos; y prestar diversos servicios para que puedan satisfacer sus necesidades de desarrollo integral.

En esta línea, Barton (2009) propone que la educación inclusiva tiene que ver con el proceso de incrementar y mantener la participación de todas las personas en la sociedad, escuela o comunidad de forma simultánea, procurando disminuir y eliminar todo tipo de procesos que lleven a la exclusión, implicando la desaparición de toda forma de discriminación, así como decidir qué necesidades deben ser atendidas y cómo.

Esta educación inclusiva, no debe reducirse solo a la enseñanza obligatoria, sino que también debe extenderse a la educación superior, tal como sostienen Sachs y Schreuer (2011), el nivel educativo tiene directa relación con las oportunidades de participación y esto, además, es un buen predictor de cómo se integren a los contextos laborales. Lo anterior es reafirmado por Jordán de Urries y Verdugo (2014), quienes indican que las personas con discapacidad tienen bajo nivel de empleabilidad, lo que debe enfrentarse, 
entre otras formas, facilitando la participación en la educación superior. A esto, Liasidou (2014) agrega que la inclusión en educación superior es parte de la promoción de la justicia social. El promover este proceso, es parte de disminuir la desventaja social experimentada históricamente por los estudiantes con discapacidad.

Si lo recién expuesto justifica la importancia de la inclusión en educación superior, aún queda conocer cómo se logra este proceso. En base a esto se hace necesario conocer cuáles son las barreras y, más aún, algo que no ha sido tan investigado: cuáles son los factores que facilitan la inclusión en este nivel educativo.

Según Padilla (2011), la discapacidad actualmente es definida en función de la interacción y participación del individuo en su ambiente, de este modo, basándose en el componente interactivo, es fundamental conocer la perspectiva no solo de los alumnos, sino también la de otros actores involucrados respecto a cuáles perciben ellos son aquellos factores que obstaculizan un adecuado proceso de inclusión y cuáles podrían facilitarlos.

Es importante agregar que Lissi et al. (2009) señalan que no cualquier institución de educación superior puede preciarse de inclusiva, pues para ello debe cumplirse una serie de condiciones. En primer lugar, se debe contar con políticas al interior de las universidades que promuevan la inclusión y que consideren medidas antidiscriminatorias, además, dentro de los aspectos transversales debe procurarse acceso a todos los programas de estudio dispuestos en la universidad y el desarrollo de actividades permanentes de sensibilización en la comunidad universitaria que promuevan la inclusión y equidad de condiciones para los estudiantes con discapacidad. Otro aspecto es que las instituciones cuenten con un censo de estudiantes, docentes y personal administrativo con discapacidad.

Considerando los antecedentes antes expuestos respecto al derecho que todas las personas tienen a acceder a la educación y la forma en que la educación mejora las oportunidades de quienes están en situación de discapacidad, el problema abordado en el presente estudio guarda relación con conocer cómo se da este proceso en una importante institución educativa como lo es la Pontificia Universidad Católica de Chile, que

Ha mostrado la diferencia entre una universidad meramente integradora, como lo era hace un tiempo atrás, en que se admitían postulantes con discapacidad sin la existencia articulada de apoyo, y una universidad que incorpora un programa como el PIANE-UC para hacerse cargo de estos apoyos. (Lissi et al., 2009, p. 322).

Lo antes expuesto es relevante para hacer las mejoras necesarias en el propio programa, y también podría ser de gran utilidad para dar lineamientos a otras instituciones de educación superior que trabajen con alumnos con algún tipo de discapacidad. Considerando lo pionera que es esta universidad en la materia, se podría contribuir así al proceso de inclusión en educación superior en Chile.

Es importante destacar que el presente estudio es parte de una tesis para optar al grado de magíster, y esta a su vez es parte de una investigación asociada al Proyecto "Sistematización de la experiencia de trabajo colaborativo entre el Piane-UC y las unidades académicas, para la implementación de adecuaciones curriculares para estudiantes con discapacidad en la UC" de las investigadoras María Rosa Lissi y María Soledad Zuzulich, del PIANE-UC, financiado por la Vicerrectoría Académica y la Pastoral de la Pontifica Universidad Católica de Chile. 
A continuación se expone un marco conceptual necesario para comprender la problemática.

\section{MARCO CONCEPTUAL}

\subsection{CONCEPTO DE DISCAPACIDAD E INCLUSIÓN}

Según Da Silva, Guilhem y Dornelles (2010) la conceptualización de la discapacidad ha variado a través de los años. Así, hay una perspectiva inicial basada en un Modelo Médico que se centraba en la enfermedad o en la limitación física como la causa principal de la desigualdad social y de las desventajas experimentadas por "las personas que presentan deficiencias", ignorando el papel de las estructuras sociales en su opresión y marginalización (Da Silva, Guilhem \& Dornelles, 2010).

Según Anastasiou y Kauffman (2011), el modelo médico se caracteriza por proponer que la causa de la discapacidad radica en el individuo y que los factores sociales no inciden mayormente. Este modelo también se asocia a crear un sistema taxonómico para categorizar las distintas discapacidades, lo que se traduce en que las personas con discapacidad sean materia de etiquetado, por otra parte, al buscar la "cura" a la discapacidad se generarían intereses creados en la industria médica. Finalmente, estos autores indican que este modelo connota una actitud profesional cruel hacia las personas con discapacidad, una relación paternalista desde el profesional que invade la privacidad de las personas (Oliver, 1990, 1993; Oliver \& Barnes, 1993 Cit. en Anastasiou \& Kauffman, 2011).

En contraposición surge el Modelo Social, donde la causa de la exclusión está en la estructura social, es decir, la deficiencia no debe ser entendida como un problema individual, sino más bien una cuestión de la vida en sociedad, lo que transfiere la responsabilidad por las desventajas de las limitaciones corporales del individuo a la incapacidad de la sociedad en prever y ajustarse a la diversidad, así

[...] no son las limitaciones individuales las raíces del problema, sino las limitaciones de la propia sociedad, para prestar servicios apropiados y para asegurar adecuadamente que las necesidades de las personas con discapacidad sean tenidas en cuenta dentro de la organización social”. (Palacios, 2008, p. 28).

Además, se establece que las personas con discapacidades aportan a la sociedad, de igual manera que cualquier otra persona, en función de la inclusión y la aceptación de la diferencia que exista en esta sociedad. Barton (2003 Cit. en Palacios 2008) agrega que la construcción social no se reduce a una problemática de origen individual, ya que la discapacidad también se puede definir como "barreras de incapacitación", que establecen las estructuras sociales, ideológicas, políticas y materiales.

Siguiendo la lógica del modelo recién planteado, es importante señalar que el término inclusión alude a "la necesidad de cambiar los sistemas de atención y apoyo no solo en el ámbito individual, sino también en el de las organizaciones y la sociedad en general" (Echeíta \& Verdugo, 2004, p. 11.)

Kioko y Mikoelle (2014) señalan que es fundamental comprender el proceso de inclusión como algo dinámico, que va cambiando de acuerdo a las necesidades que 
van surgiendo, por lo que es trascendental estar atentos permanentemente a lo que van expresando los actores involucrados.

\subsection{BARRERAS Y FACILITADORES}

Muccio (2012) indica que el proceso de inclusión se vería influido por ciertas variables. Aquellas que impiden o inhiben la participación de los alumnos se denominan barreras, y aquellas que hacen exitosa la implementación de la inclusión se les llama facilitadores.

\subsubsection{Barreras}

Darrow (2009) clasifica las barreras en tres áreas: organizacional, actitudinal y de conocimiento. Las primeras son las referentes a la forma en que las instituciones y las clases son estructuradas, cómo se definen los objetivos propuestos para los alumnos con discapacidad, cómo son las estrategias de enseñanza y cómo son manejadas las clases.

Las barreras de tipo actitudinal se relacionan con las creencias y actitudes que los profesores puedan tener sobre los servicios educativos para los estudiantes con discapacidad, incluidos los ajustes curriculares, las interacciones con los estudiantes, los estudiantes, la participación en la institución y actividades de la comunidad.

Las actitudes negativas pueden derivarse de la falta de información, experiencias previas y las situaciones difíciles que siguen sin resolverse o sin éxito. Los profesores pueden tener ideas erróneas sobre el trabajo con los estudiantes que tienen discapacidades o pueden tener el temor de que no van a ser maestros eficaces en un ambiente inclusivo. Los docentes también pueden estar preocupados por la forma en que la inclusión afectará el clima del aula y la educación de los estudiantes que no tienen discapacidades.

Sin embargo, según Darrow (2009), las actitudes positivas pueden desarrollarse y mejorarse de muchas maneras diferentes, por ejemplo, animando a los profesores a descubrir las fortalezas de los estudiantes y el desarrollo de métodos de enseñanza y las adaptaciones que se basan en los puntos fuertes.

Finalmente, las barreras referentes a los conocimientos se refieren a "la gama de conocimientos y habilidades que necesitan los profesores a fin de proporcionar servicios eficaces a los estudiantes, como la adaptación del plan de estudios y métodos de enseñanza" (Darrow, 2009, p. 30).

Respecto a las barreras, Sánchez (2010) agrega que estas "se generan desde la planificación, desarrollo y evaluación de los proyectos curriculares en las adaptaciones de objetivos, contenidos, organización de apoyos y la forma de entender la evaluación en el proceso de enseñanza aprendizaje".

Por su parte Borland y James (1999) distinguen barreras de acceso (infraestructura y espacios) y barreras de acceso al currículo, referidas a aquellos aspectos que dificultan la participación en el espacio de enseñanza-aprendizaje. Además, según Lissi et al. (2009) es posible agregar una tercera clase de barreras, que tiene que ver con aspectos más generales como la falta de compromiso o de cooperación de los docentes hacia las adecuaciones.

En tanto, Konur (2006) concuerda en que las principales barreras con las que se encuentran estos estudiantes al acceder a la educación superior pueden ser clasificadas en problemas de acceso a los programas y al currículum, a su vez, Katsiyannis y otros (2009) 
agregan que estas barreras dan cuenta de falta de conocimiento tanto de las discapacidades como de las leyes que amparan la inclusión y los ajustes necesarios para cada caso.

Más cercano al contexto de esta investigación, Medrano (2009) en un estudio cualitativo sobre la inclusión en la Pontificia Universidad Católica y otras instituciones de educación superior chilenas, encontró como barreras la falta de información de los docentes, dificultades de los propios estudiantes para enfrentar las exigencias académicas de la universidad, problemas con la infraestructura y, finalmente, se reconocen algunas dificultades asociadas a sus discapacidades específicas.

\subsubsection{Facilitadores}

Si bien hay bastantes investigaciones que aluden a las barreras, disminuyen aquellas enfocadas en los facilitadores de la inclusión, y las que se han desarrollado, en general, se restringen al nivel escolar o al contexto personal del alumno.

Dentro de los estudios referentes al ámbito escolar, Pivik, McComas y Laflamme (2002) identifican como facilitadores tres aspectos a abordar: modificaciones ambientales, cambios en las políticas y recursos institucionales. En cuanto a las modificaciones ambientales consideran importante incluir recursos tecnológicos y adecuar la infraestructura a las necesidades de los alumnos y respecto a las políticas, recomiendan educar a la población y realizar adecuaciones curriculares.

Referente al contexto personal del alumno se menciona la existencia de soportes sociales y del apoyo de la familia o la resiliencia que se relacionaría con la capacidad de adaptarse a la discapacidad (Frain et al., 2007 Cit. en Medrano, 2009), factores que por cierto solo se pueden desarrollar personalmente en cada alumno. Es así como hay poca literatura que se aboque a aquellos que son propios del contexto educacional universitario, y menos aún se enfocan específicamente a integrar las percepciones de los propios alumnos y de los equipos docentes.

\subsection{PROGRAMA PARA LA INCLUSIÓN DE ALUMNOS CON NECESIDADES ESPECIALES (PIANE-UC)}

Basándose en lo anteriormente expuesto, en la Pontificia Universidad Católica de Chile, el 2006, surge el PIANE-UC, cuyo objetivo es

[...] promover la inclusión de estos alumnos, buscando otorgar condiciones de equidad e igualdad de oportunidades para los estudiantes que presentan necesidades educativas especiales (NEE) asociadas a discapacidad sensorial o motora, en todos los ámbitos del quehacer académico y de la vida estudiantil, con el propósito de ir generando una cultura cada vez más inclusiva en nuestra universidad. (Lissi et al., 2013, p. 61).

Así, este programa brinda apoyo a los estudiantes con discapacidad en aspectos académicos y tecnológicos, también asesoría a los docentes en adecuaciones curriculares y capacitación en el uso de recursos tecnológicos especializados, además procura sensibilizar a la comunidad universitaria respecto del proceso de inclusión de alumnos con discapacidad y genera investigación en el área de la inclusión en la Educación Superior (Lissi et al., 2013).

Dentro de las acciones específicas para los estudiantes se realizan "tutorías de pares", que consisten en que alumnos de las distintas carreras apoyan el desempeño académico de 
los estudiantes con discapacidad, mediante apoyo académico directo al alumno, orientación, adaptación del material y mediación con los docentes.

Lissi et al. (2013) plantean que mediante este programa además se brinda apoyo a docentes, a través de la entrega de información, dando orientaciones generales respecto a que tendrán a un estudiante con discapacidad; asesorías mediante las que pueden resolver dudas puntuales con relación a cómo llevar a cabo las adecuaciones curriculares apropiadas a su curso; talleres o reuniones informativas; capacitación a ayudantes y adecuación del material pedagógico.

\subsection{TUTORÍA DE PARES}

Según Vogel, Fresko y Wertheim (2007) la tutoría de pares en contextos educativos ha mostrado ser una estrategia efectiva que favorece la inclusión de estudiantes con discapacidad y dificultades de aprendizaje. Esta estrategia beneficia tanto a los alumnos tutorados como a los propios tutores. Respecto a los primeros, mejora su rendimiento académico y su adaptación social (Vogel et al., 2007), además previene la deserción en el ámbito de la educación superior (Kowalsky \& Fresko, 2002). En relación a los segundos, se promueve un mayor conocimiento respecto a las personas con discapacidad, el aumento de expectativas y actitudes más positivas hacia estas personas, la experimentación de sentimientos de éxito, mayores oportunidades de interacción social y el aumento de habilidades académicas (Lissi \& Onetto, 2012).

Las tutorías de pares implementadas en la UC consisten en que las distintas unidades académicas contratan estudiantes para que sean tutores pares de los alumnos con discapacidad. Estos son capacitados por el PIANE-UC y también se les brinda seguimiento y apoyo. El foco de este apoyo es el proceso académico; el tutor es un mediador entre el estudiante con discapacidad y sus docentes, para apoyar la implementación de las adecuaciones necesarias (Lissi et al., 2012).

\section{MATERIAL Y MÉTODO}

El material utilizado para esta investigación estuvo constituido por 9 entrevistas realizadas a los tutores participantes del programa PIANE-UC. Los participantes fueron seleccionados considerando la importante labor que realizan en el PIANE UC e intentando abarcar distintas disciplinas y distintos tipos de discapacidad. Así, los tutores entrevistados pertenecían a las carreras de Sociología, Agronomía, Letras Hispánicas, Historia, Trabajo social, Letras Inglesas, Psicología e Ingeniería (dos tutores de esta última carrera) y apoyaban a alumnos con discapacidad de tipo visual (5 alumnos), motora (4 alumnos) y auditiva (1 alumno).

Las entrevistas antes descritas se analizaron desde la Teoría Fundamentada. Esta metodología fue desarrollada por los sociólogos Glasser y Strauss en 1967, con gran influencia del Interaccionismo Simbólico. Este influjo se refleja en la necesidad de hacer estudios de campo para comprender la realidad y en comprender que el significado de la realidad cambia y se redefine en función de las interacciones.

De este modo, la Teoría Fundamentada utiliza un procedimiento sistemático cualitativo para generar una teoría que explique en un nivel conceptual una acción, una interacción o un área específica. Esta teoría es denominada sustantiva o de rango medio y se aplica a un 
contexto más concreto. Glaser y Strauss (1967) distinguen este tipo de teoría de la "teoría formal", cuya perspectiva es mayor. Las teorías sustantivas son de naturaleza "local" (se relacionan con una situación y un contexto particular). Sus explicaciones se circunscriben a un ámbito determinado, pero poseen riqueza interpretativa y aportan nuevas visiones de un fenómeno. Es decir, esta metodología tiene como objetivo desarrollar teoría a partir de una sistemática recogida y análisis de datos, llegando a un esquema analítico abstracto de un fenómeno que se relaciona con una situación y un contexto particular (Sandín, 2003).

La teoría fundamentada se desarrolla en tres fases: Codificación abierta, codificación axial y codificación selectiva, con el fin de generar un modelo comprensivo acerca del fenómeno en estudio.

La codificación abierta es el primer paso, es el proceso analítico por medio del cual se identifican conceptos y se establecen categorías sobre un fenómeno en particular (Strauss $\&$ Corbin, 2002), las que tienen propiedades y dimensiones.

La codificación axial es el proceso de relacionar categorías y subcategorías entre sí para comprender cuáles son los contextos, las condiciones o causas, las estrategias y las consecuencias que están presentes en el fenómeno (Strauss \& Corbin, 2002). Hernández, Fernández y Baptista (2010) especifican que las condiciones causales son aquellas categorías que influyen y afectan a la categoría central; las acciones e interacciones son categorías que resultan de la categoría central y las condiciones contextuales e intervinientes, así como de las estrategias. Las consecuencias son categorías resultantes de las acciones e interacciones y del empleo de las estrategias, las que a su vez son categorías de implementación de acciones que influyen en la categoría central y en las acciones, interacciones y consecuencias. Las condiciones contextuales son categorías que forman parte del ambiente o situación y que enmarcan a la categoría central, que pueden influir en cualquier categoría, incluyendo a la principal. Finalmente, las condiciones intervinientes son categorías que también influyen a otras y que mediatizan la relación entre las condiciones causales, las estrategias, la categoría central, las acciones e interacciones y las consecuencias. Es importante aclarar que el presente estudio alcanzó hasta este nivel de análisis.

El paso final es la codificación selectiva, que es el proceso donde se refina y se integra la teoría alrededor de un concepto explicativo central (Strauss \& Corbin, 2002).

La información obtenida de las entrevistas se trianguló con tres profesionales candidatos a magíster en psicología educacional, con experiencia en el área de inclusión y conocimientos en metodología cualitativa.

\section{RESULTADOS}

Los tutores entrevistados dieron cuenta de ocho grandes temáticas asociadas al proceso de inclusión en la Pontificia Universidad Católica. Destacando como fenómeno central las prácticas inclusivas o excluyentes de los equipos docentes, las que promovían o dificultaban la participación en equidad de condiciones de los alumnos con discapacidad. Según los entrevistados, este fenómeno es antecedido y fuertemente influido por la conceptualización que los actores tengan de la discapacidad y se ve reflejado en cómo se implementa el ramo y la forma en que la comunidad educativa y los alumnos con discapacidad se relacionan. Además, en las entrevistas se destaca el contexto como un factor que influye directamente en el fenómeno, aludiendo principalmente a las políticas de la universidad. 
Lo antes expuesto fue extraído del análisis realizado a las entrevistas utilizando la Teoría Fundamentada. A continuación, se exponen más detalladamente las categorías, propiedades y dimensiones encontradas (Cuadro 1).

Cuadro 1. Descripción de categorías

\begin{tabular}{|c|c|}
\hline Categorías & Propiedades y Dimensiones \\
\hline $\begin{array}{l}\text { I. } \\
\text { Prácticas de los equipos docentes }\end{array}$ & $\begin{array}{l}\text { Propiedades: } \\
\text { - Adecuaciones. } \\
\text { - Participación de los alumnos con discapacidad. } \\
\text { Dimensiones: } \\
\text { - Prácticas excluyentes. } \\
\text { - Prácticas inclusivas. }\end{array}$ \\
\hline $\begin{array}{l}\text { II. } \\
\text { Relación con la comunidad educativa }\end{array}$ & $\begin{array}{l}\text { Propiedades: } \\
\text { - Disposición de los pares. } \\
\text { - Vínculo con los pares. } \\
\text { - Conductas proactivas de los alumnos. } \\
\text { Dimensiones: } \\
\text { - Relaciones Cercanas con la comunidad. } \\
\text { - Relaciones Distantes con la comunidad. }\end{array}$ \\
\hline $\begin{array}{l}\text { III. } \\
\text { Implementación del ramo }\end{array}$ & $\begin{array}{l}\text { Propiedades: } \\
\text { - Actividades fuera del aula. } \\
\text { - Contenidos de difícil acceso. } \\
\text { - Práctica profesional. } \\
\text { Dimensiones: } \\
\text { - Permiten la participación del alumno. } \\
\text { - Dificultan la participación del alumno. }\end{array}$ \\
\hline $\begin{array}{l}\text { IV. } \\
\text { Adecuaciones }\end{array}$ & $\begin{array}{l}\text { Propiedades: } \\
\text { - Adecuaciones de material. } \\
\text { - Adecuaciones en evaluaciones. } \\
\text { Dimensiones: } \\
\text { - Adecuaciones No significativas. } \\
\text { - Adecuaciones significativas. }\end{array}$ \\
\hline
\end{tabular}




\begin{tabular}{|c|c|}
\hline $\begin{array}{l}\text { V. } \\
\text { Apoyos }\end{array}$ & $\begin{array}{l}\text { Propiedades: } \\
\text { - Apoyos de los tutores. } \\
\text { - Recursos Tecnológicos. } \\
\text { - Infraestructura. } \\
\text { Dimensiones: } \\
\text { - Apoyos favorecen la participación de los alumnos con } \\
\text { discapacidad. } \\
\text { - Apoyos no inciden en la participación de los alumnos } \\
\text { con discapacidad. }\end{array}$ \\
\hline $\begin{array}{l}\text { VI. } \\
\text { Políticas de la universidad }\end{array}$ & $\begin{array}{l}\text { Propiedades: } \\
\text { - Responsabilización de las escuelas. } \\
\text { - Institucionalización de las prácticas inclusivas. } \\
\text { Dimensiones: } \\
\text { - Políticas que se implementan en la institución. } \\
\text { - Políticas que aún no se implementan en la institución. }\end{array}$ \\
\hline $\begin{array}{l}\text { VII } \\
\text { Conceptualización de la discapacidad }\end{array}$ & $\begin{array}{l}\text { Propiedades: } \\
\text { - Conocimientos sobre la discapacidad. } \\
\text { - Accesibilidad a capacitación. } \\
\text { - Experiencia previa con personas con discapacidad. } \\
\text { - Sensibilización sobre la discapacidad. } \\
\text { - Visibilidad de la discapacidad. } \\
\text { Dimensiones: } \\
\text { - Conceptualización desde un modelo médico. } \\
\text { - Conceptualización desde un modelo social. }\end{array}$ \\
\hline $\begin{array}{l}\text { VIII. } \\
\text { Presencia de un programa articulador }\end{array}$ & $\begin{array}{l}\text { Propiedades: } \\
\text { - PIANE como mediador. } \\
\text { - Difusión del PIANE. } \\
\text { Dimensiones: } \\
\text { - Programa favorece la participación de los alumnos con } \\
\text { discapacidad. } \\
\text { - Programa no influye en la participación de los alumnos } \\
\text { con discapacidad. }\end{array}$ \\
\hline
\end{tabular}


En función de estas categorías y sus relaciones se propone el siguiente modelo general:

Figura 1. Modelo general

Contexto: Políticas de la Universidad

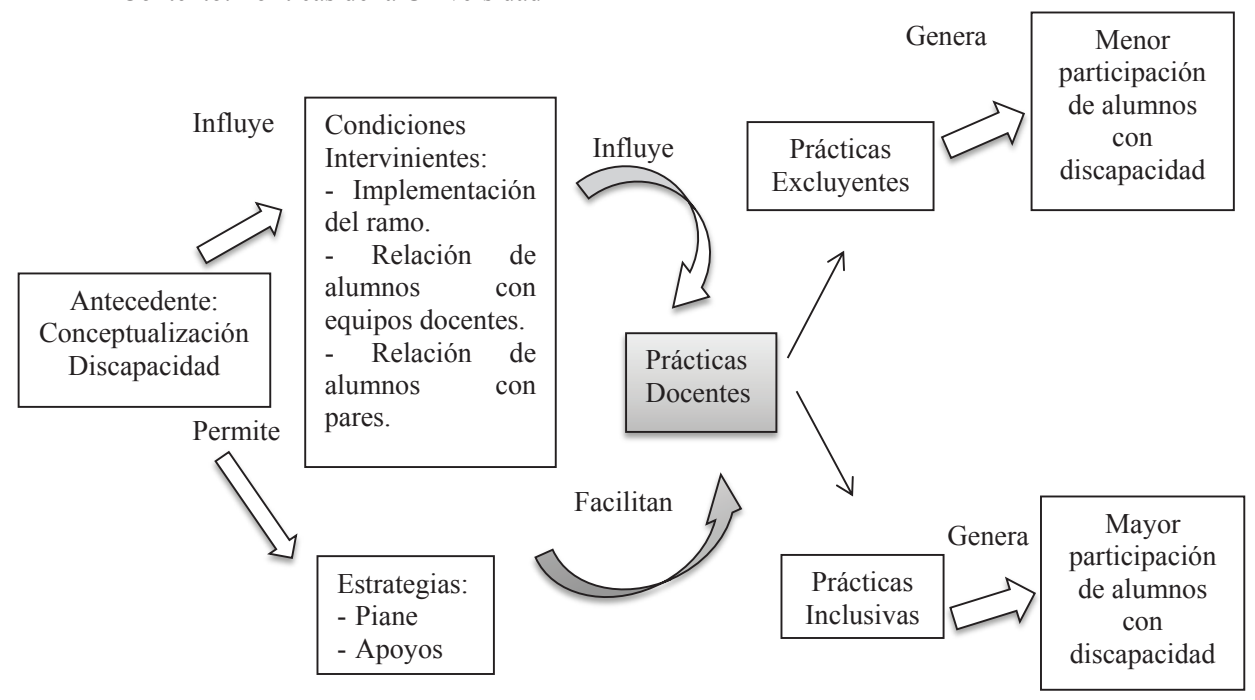

En base a la Teoría Fundamentada, los entrevistados destacan el fenómeno prácticas docentes, distinguiendo entre aquellas que son inclusivas y generan mayor participación de alumnos con discapacidad y aquellas excluyentes y que obstaculizan la participación de estos alumnos.

Ante este fenómeno, es posible señalar, como antecedente principal, la forma en que los distintos actores conceptualizan la discapacidad, ya sea desde un modelo médico, centrado en las deficiencias de la persona, o de un modelo social, enfocado en las barreras que genera la sociedad y que influye en algunas condiciones intervinientes como la forma en que se implementan los distintos ramos.

También esta conceptualización influye en otra condición interviniente, que es la relación de los alumnos con discapacidad con los equipos docentes, tanto por la disposición que presentan docentes y ayudantes como por la proactividad del mismo estudiante. Si hay una visión más médica, los equipos tendrán menos disposición a favorecer un aprendizaje en equidad de condiciones, centrándose en los déficits de los alumnos como algo que solo se puede abordar facilitándoles las cosas. Por otra parte, si son los mismos estudiantes los que se posicionan desde una visión más médica, serán menos proactivos, pues visualizarán la discapacidad como una enfermedad contra la que no se puede hacer mucho, victimizándose.

Cabe señalar que los tutores entrevistados perciben que los pares de los alumnos también son un elemento importante a considerar en el proceso de inclusión. Su disposición a trabajar con sus compañeros con discapacidad y el vínculo que establecen con ellos afectan a la inclusión de los alumnos con discapacidad. 
La conceptualización que la universidad ha tomado respecto a la discapacidad, guarda directa relación con las estrategias que se han implementado, los apoyos de distinto tipo y la presencia de un programa articulador.

Por otra parte, como contexto, las políticas de la P. Universidad Católica, el modo en que se ha ido transitando desde un modelo más médico a uno social han permitido que se implementen como estrategias la presencia de un programa (PIANE) y se entreguen apoyos de distinto tipo, lo que facilita que las prácticas sean inclusivas, es decir que mediante adecuaciones que no modifican los objetivos de los ramos facilitan y promueven la participación en clases de los alumnos con discapacidad:

T1: "No era que tuviéramos un material especial, por ejemplo, o que tuviéramos que, no sé, venir acá y que le tuvieran que pasar todo a braille, por ejemplo. Nosotros trabajábamos con el mismo material que se entregaba en la clase, que por lo general son presentaciones en power point y ella se preocupaba de imprimirlas grande”.

Las barreras son aquellas que obstaculizan dicha participación, no realizando adecuaciones o ejecutando cambios sustanciales en los objetivos del ramo.

T2: "hubo profes que les complicaba más el tema de tener que tomarle una prueba a un alumno solo, porque después decían que: Ay! Les pueden dar las respuestas ¡ay que esto!, ;ay que esto otro!, si al final era cosa de honestidad hacia la misma persona y también al resto de los compañeros ya pasa por otro tema valórico que ellos no pueden hacer nada en ese sentido".

A continuación, se profundizará en algunos ejes del modelo recién expuesto:

\subsection{RELACIÓN ENTRE LOS ALUMNOS CON DISCAPACIDAD Y LOS EQUIPOS DOCENTES}

Figura 2. Relación entre estudiantes con discapacidad y equipos docentes

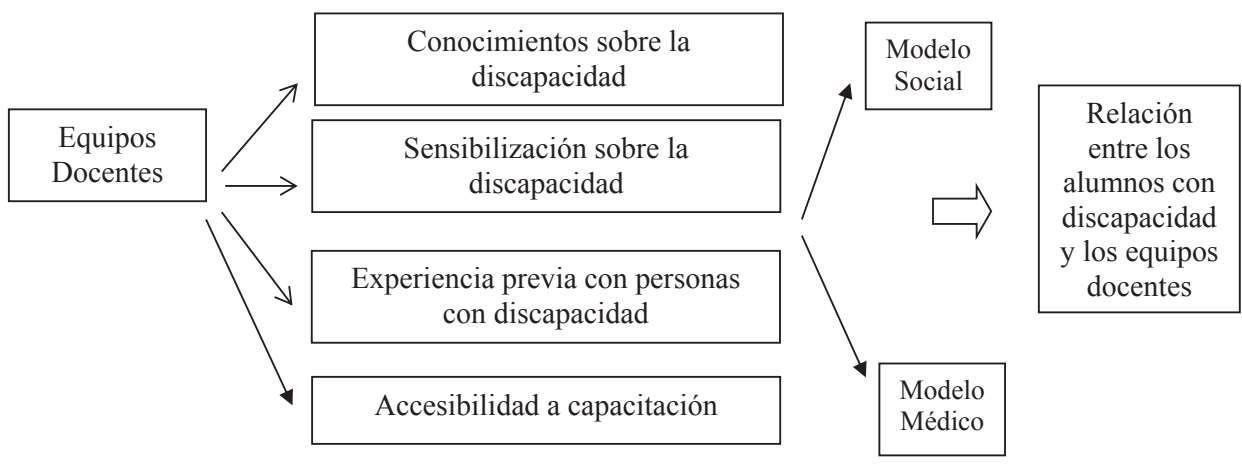


De acuerdo al marco teórico explicitado previamente hay dos modelos centrales desde los cuales tanto los propios estudiantes con discapacidad como los equipos docentes abordan la discapacidad: el social y el médico. En función de los conocimientos que se tengan, la experiencia previa, la accesibilidad a capacitación y la sensibilización que se logre respecto a la discapacidad, se tendrá una visión más médica o social, que condicionaría la disposición y la relación que se establezca con los pares y docentes.

Cabe aclarar que el concepto de sensibilización hace referencia a concientización de los actores respecto a las características, necesidades y demandas de los alumnos con discapacidad. La información que tengan los actores respecto a la discapacidad, las características de estos alumnos, sus necesidades y cómo responder a ellas, influye en la visión que tengan de la discapacidad y sus prácticas.

T2: "como que se ve eso es como secreto a voces entre comillas, pero es como la cultura inculta que cree que son personas diferentes por tener una habilidad diferente no más, que no saben asumir esa diferencia que, porque igual uno puede ser más seco para matemáticas y al otro lado mejor en ciencias, no entienden que esa habilidad diferente es la misma que tienen ellos solamente que se refleja de forma física".

La experiencia previa permite que los actores visualicen empíricamente cuáles son las reales necesidades de un alumno con discapacidad y paulatinamente van experimentando cómo dar respuesta a ellas:

T2: "y yo decía pucha a mi mamá le hubiera tocado algo así en el colegio, o si le hubiese tocado en el trabajo programas de inclusión así, o a mis primos, como que ese tema como que igual uno le llega mucho más cuando uno lo ha vivido con gente cercana".

También los tutores plantean como una necesidad el sensibilizar respecto a la discapacidad, argumentándose que con esto se visualizará el fenómeno como algo social, más que como una característica personal de los alumnos.

T5: "La gente quiere, compañeras mías quieren, están abiertas a conocer, pero no están los minutos, no hay dónde. De repente acercarse dentro de la misma unidad pedagógica, dentro de la misma escuela de trabajo social, esa energía de decir sabes que nuestros alumnos deben saber comunicarse con quien sea, lenguaje de señas, lenguaje braille, algo, pero que se hagan más instancias para conocer, tienen que hacer más promoción, no solo en panfletos chiquititos, sino que además extras, va gente con necesidades especiales, pero también podrían trabajar en esa instancia con personas que se quieran formar para saber cómo enfrentarla, encuentro que esa es como el corazón, porque no nos sirve de nada estar trabajando con un granito que está siendo atacado por toda una sociedad que la discrimina, si no trabajamos con la sociedad”. 


\subsection{IMPLEMENTACIÓN DEL RAMO}

Figura 3. Implementación de las asignaturas

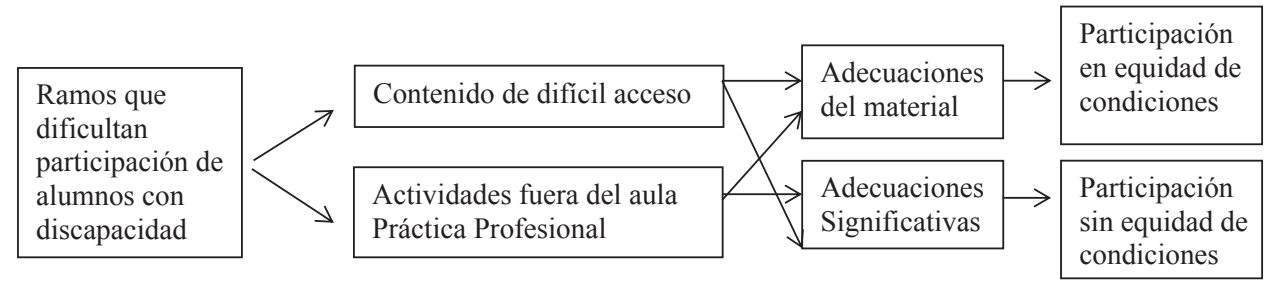

Según lo reportado por los tutores entrevistados, existen ciertas asignaturas que presentan un mayor desafío en cuanto a las prácticas inclusivas, ya sea porque el contenido en sí es de difícil acceso, especialmente para quienes presentan discapacidad visual, como aquellos ramos más biológicos o matemáticos, que tienen mucha información de este tipo, o porque hay actividades que significan en sí un obstáculo.

T2: “Nosotros nos hicieron subir al valle del Elqui, ¡pero una pendiente así! Y era sobre el 100\% o sea tenía más de 45, o sea estaba a 70 grados de empinada la cuestión, y nos hicieron subirla, o sea y después de subirla nosotros decíamos, nosotros que podemos ocupar bien las manos, los pies todo eso nos costó pero un kilo subir esa cuestión, o sea yo decía esa salida no se las podí hacer a todos los alumnos, entonces tení que tener esa flexibilidad que el alumno aprenda y todo, pero no puede hacer las mismas actividades que uno o sea; queraí o no pucha es verdad o sea él puede aprender, el mismo tema pero aprenderlo de otra forma, quizás viendo como sudamos la gota gorda nosotros que si podemos subir jajaja, y va a cachar que es terrible subir esa pendiente, pero no tienes para que hacerle subir a él”.

En el caso de contenido de difícil acceso, los actores señalan que al hacer adecuaciones del material se logra la inclusión de los alumnos con discapacidad. El problema surge en aquellas actividades prácticas, fuera del aula, donde quienes tienen un trastorno motor o visual no pueden participar, perdiendo importantes aprendizajes o en la práctica profesional donde, como refieren los entrevistados, el contexto no está adecuado a las necesidades de los alumnos con discapacidad, y se les hace muy difícil pensar en una adecuación que no sea significativa:

T2: “Tal vez para los pasos prácticos por ejemplo si ella tuviera que desplazarse e ir a la escuela de La Pintana por decirte que es donde se asignan los casos y ahí no hay ramplas, no hay... la sala que le prestan está en el segundo piso y no hay como subir al segundo piso, ahí yo creo que sí, y efectivamente si uno va más a la periferia sí ya hay poco en Santiago: ramplas, bajadas, subidas y accesos para personas que están en sillas, si uno va a La Pintana nada". 
FACILITADORES Y BARRERAS DEL PROCESO DE INCLUSIÓN EN EDUCACIÓN SUPERIOR: LA PERCEPCIÓN DE LOS TUTORES DEL PROGRAMA PIANE-UC

\subsection{RELACIÓN CON LOS PARES}

Figura 4. Relación con pares y participación de estudiantes con discapacidad

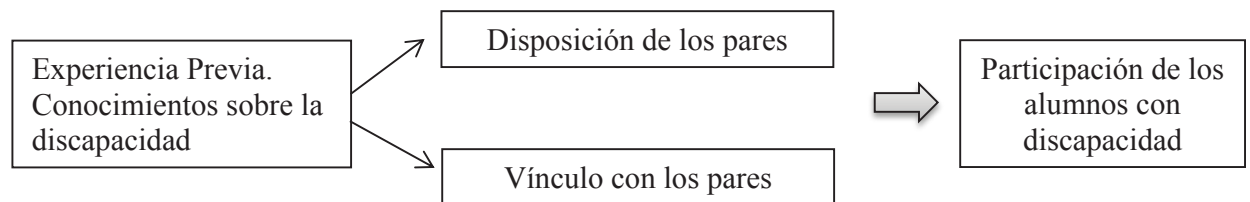

La experiencia y los conocimientos que los compañeros de los alumnos con discapacidad tienen sobre esta, afecta a la disposición y al vínculo que establecen con sus pares. Los tutores señalan que cuando hay desconocimiento sobre las capacidades de los alumnos con discapacidad, se tiende a excluirlos, y, por el contrario, hay una mejor disposición cuando tienen otra visión de ellos, lo que favorece que se genere la inclusión.

T3: "hay mucho desconocimiento entonces es como ah no, no trabajemos con este porque es, muy lenta, porque es muy tonta”.

Por otra parte, se reporta que el apoyo sostenido de los compañeros cuando establecen un vínculo, también sería un facilitador, por el contrario, cuando no se establecen estos lazos hay menos posibilidad de participación de los estudiantes con discapacidad.

T1: "La AL nunca tuvo tanta relación con su generación. Nada que ver, era una generación chica, yo la encuentro que, por el tipo de gente, tenía amigos en otras carreras, como que nunca formó mucha relación y eso yo creo que también en alguna medida te puede... no sé si jugar en contra, pero en el fondo puede ser un facilitador para otras cosas".

\subsection{CONTEXTO}

Figura 5. Contexto

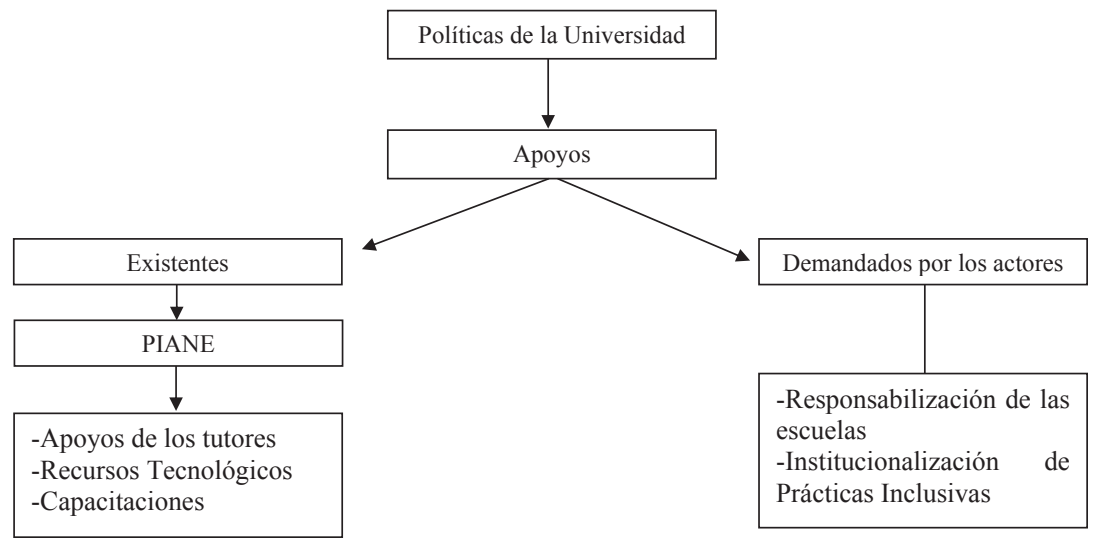


El contexto en el que están los entrevistados es un importante factor a considerar, según lo planteado por ellos mismos, haciendo referencia a las políticas de la universidad. En este aspecto se identifican dos líneas. Por un lado, las políticas entregan ciertos apoyos, principalmente mediante el PIANE, que es visualizado como un facilitador y una ayuda tanto para los alumnos como para los equipos docentes (capacitaciones), siendo también un mediador entre ambos estamentos.

T1: "Entonces también ahí, veo yo que la labor como institucional del PIANE en relación a los profesores, como ser un nexo entre profesor y alumno. No es lo mismo que se te acerque un alumno a decirte oiga profesor tengo esto y esto otro, porque los profesores están tan incrédulos de todo".

La principal labor atribuida a este programa son los apoyos que se entregan a los alumnos tanto de tipo humano (tutores) como material (recursos tecnológicos), los que son un gran facilitador de la participación de los alumnos con discapacidad en igualdad de condiciones que sus pares.

T2: "lo que pasa es que AL tiene un programa que se llama Jaws donde le va leyendo los caracteres, y él puede como retrogradar el Jaws para que le diga ciertas cosas cuando aparecen ciertos caracteres".

Por otra parte, hay ciertas demandas por parte de los actores sobre acciones que la universidad, dentro de sus políticas, debiera promover. En primer lugar, que las escuelas se responsabilicen por las necesidades de sus alumnos con discapacidad, y también que las prácticas inclusivas sean algo institucional, no optativo.

T3: "el otro facilitador era la unidad académica, que en el fondo brindó mucho apoyo pagándole a estas otras personas para que pudieran escanear porque yo no sé cómo lo hubiese hecho escaneando esa cantidad de textos, porque en historia se lee muchísimo, como 600 páginas a la semana, entonces yo escaneando 600 páginas a la semana no me veía mucho entonces, la unidad académica fue facilitador enorme".

T2: "no sé si del reglamento interno de la facultad, pero que a ese alumno se le tiene que tratar de forma igual y va a estar ese como opcional entre comillas, pero que va a ser esa vía para él, como que lo estipulen; hay muchos profes como que por voluntad que lo hacen y que ya no sea como voluntad si no que es así, es un programa de inclusión y la universidad lo tiene asumido como algo de inclusión, entonces tiene que estar estipulado, no es como la voluntad ni a la suerte de la olla, sino porque lo tienen que hacer, que corresponde o sea es lo éticamente correcto, es lo mínimo que se puede hacer".

\section{CONCLUSIONES Y DISCUSIÓN}

La inclusión en educación superior es un fenómeno complejo en el que participan varios actores y que es influido por una serie de elementos.

Según lo reportado por los participantes considerados en este estudio, un elemento fundamental lo constituye la conceptualización que tiene la comunidad universitaria 
respecto a la discapacidad, dependiendo de la visión que tengan, serán las prácticas que implementen. Pero no solo los docentes serían influidos por estos modelos, sino que también los propios estudiantes con discapacidad, quienes al asumir una visión más médica, centrada en el déficit, serían menos proactivos que aquellos que tienen una mirada más social. Esto concuerda con el análisis que realiza Liasidou (2014), quien señala que la marginación de las personas con discapacidad se ha justificado sobre la base de sus supuestas deficiencias biológicas, como resultado directo del predominio de la perspectiva de que la discapacidad es producto de una patología individual.

Esta conceptualización de la discapacidad se vería afectada por la experiencia previa que se tenga con personas con discapacidad, los conocimientos sobre la discapacidad, la accesibilidad a capacitación y la sensibilización sobre la discapacidad. De esto se desprende que un facilitador de las prácticas inclusivas es sensibilizar a la población y capacitarla respecto a lo que significa la discapacidad y cómo se pueden eliminar las barreras; esto concuerda con lo que concluyó Liasidou (2014) en su análisis, donde señala que se hace necesario sensibilizar a la población, darle "voz" a los estudiantes con discapacidad, permitiéndoles "expresar sus pensamientos y puntos de vista en relación con el enfoque, dirección y objetivos de una agenda de reforma de la justicia social en la educación superior" (Liasidou, 2014, p. 133).

Otro hallazgo relevante es la importancia que tiene la relación con los pares. Según los actores entrevistados, el apoyo de los compañeros favorecería la participación de los alumnos con discapacidad, lo que se relaciona con lo encontrado por Medrano (2009), quien señala que, desde la etapa escolar, los alumnos con discapacidad valoran el apoyo de sus pares como un facilitador de su inclusión en el contexto académico.

Pese a que el objetivo del estudio era identificar facilitadores y barreras, los participantes de esta investigación aludieron más directamente a facilitadores: una conceptualización de la discapacidad desde el modelo social, la experiencia previa que tenga la comunidad educativa con personas con discapacidad y una buena relación con los pares.

Sin embargo, es posible inferir, como principal obstaculizador, el desconocimiento respecto a la discapacidad. Cuando no se sensibiliza a la población, no hay capacitaciones o la accesibilidad a ellas es restringida se estaría dificultando que la comunidad educativa promoviera la participación en el proceso educativo de los alumnos en situación de discapacidad. Esto concuerda con los hallazgos de Kioko y Makoelle (2014) que sugieren que la educación inclusiva en el contexto de la educación superior es influenciada por una complejidad de creencias e implica toda una serie de factores, lo que genera la necesidad de una reflexión continua entre todos los miembros de la comunidad educativa.

Los resultados logrados en esta investigación permiten orientar algunas sugerencias a instituciones de Educación Superior y también al PIANE.

Teniendo en cuenta la percepción positiva que tienen los actores del programa PIANE, es posible señalar que esta es una buena iniciativa que promueve y articula la inclusión en esta institución, entregando apoyos a los alumnos con discapacidad y sirviendo como mediadora entre los alumnos y los docentes. Por ello, se sugiere que, en cuanto su particular contexto se los permita, las instituciones de Educación Superior generen un programa que les posibilite cumplir estos mismos objetivos, o tal como propusieron Lissi et al. (2009) les permita materializar el apoyo a los alumnos con discapacidad a través de la existencia de un centro dispuesto físicamente dentro de la universidad, ya que la existencia de este es crucial en el proceso de inclusión (Lissi et al. 2013) Por otra parte, dentro de los apoyos 
que entrega este programa, un recurso muy valorado por los alumnos y por los docentes es el apoyo que brindan los tutores, tanto en el ámbito académico como emocional. Iniciativa que también debería replicarse para favorecer el proceso de inclusión de alumnos con discapacidad en otras instituciones, ya que tal como señalan Lissi et al. (2012) esto no solo ayuda a los alumnos con discapacidad, sino que además representa un aprendizaje y un cambio de creencias (a favor de la inclusión) en los tutores.

En función de lo recabado en las entrevistas y los análisis realizados, también es posible entregar algunas sugerencias a la Pontificia Universidad Católica de Chile y al PIANE.

En términos generales, los actores consideraron importante que se institucionalicen las prácticas inclusivas, es decir que no se restrinjan a las labores del PIANE, sino que las diversas escuelas se involucren en la promoción e implementación de prácticas inclusivas.

En base al estudio que se presenta, como sugerencias al PIANE se pueden plantear el que realicen mejoras en cuanto a los mecanismos de difusión, ya que los docentes entrevistados señalan algunas falencias que dificultan la comunicación. También sería importante que dieran especial énfasis a la sensibilización a la comunidad educativa mediante la difusión del modelo social, permitiendo que así se involucrara a todos en el proceso de inclusión, tal como ya lo plantearon Lissi et al. (2009), destacando la importancia de realizar sensibilización, difusión y capacitación a los miembros de la comunidad universitaria que ayuden a superar las barreras para la inclusión.

Es necesario señalar que el presente estudio se abocó al proceso inclusivo en el aspecto netamente académico, lo que podría ser reduccionista, teniendo en consideración que la vida universitaria incluye también las relaciones sociales que se establezcan, la participación en grupos deportivos, políticos, religiosos, otras actividades extraprogramáticas, entre otros. En una futura investigación, sería enriquecedor conocer cómo se da la inclusión en este ámbito más allá de lo exclusivamente académico, si hay adecuaciones para que participen en actividades extracurriculares o si la infraestructura es la adecuada para que todos puedan acceder a todos los espacios, ya que tal como lo plantean Sachs y Schreuer (2011, p. 52) "La mayoría de la investigación y debate sobre la inclusión de los estudiantes con discapacidad se ha centrado en sus logros académicos, y ha descuidado las implicaciones en su participación social".

Otra limitación que se puede señalar respecto a esta investigación es que no se consideraron ciertos actores clave en el proceso de inclusión. Por ejemplo, haber contado con la percepción de algún integrante del equipo de PIANE o de algún directivo de escuela o autoridad de la universidad habría enriquecido aún más los resultados, por lo que se sugiere que en un próximo estudio puedan ser partícipes también.

Finalmente, es preciso reiterar que este estudio se realizó en una universidad específica, que tiene un programa - el PIANE-UC — con características particulares, lo que pudiera ser una limitación al intentar generalizar los resultados. Sin embargo, al tratarse de un programa pionero y ser una de las universidades más importantes del país, es una buena aproximación para guiar otros programas que se pudieran desarrollar a futuro o se estén desarrollando actualmente.

\section{REFERENCIAS BIBLIOGRÁFICAS}

Abad, M., Álvarez, P., \& Castro de Paz, J. (2008). Apoyo a la integración de estudiantes con discapacidad en la enseñanza universitaria. Educación y Diversidad, 2(1), 129-150. 
Estudios Pedagógicos XLIII, $\mathrm{N}^{\circ}$ 1: 349-369, 2017

FACILITADORES Y BARRERAS DEL PROCESO DE INCLUSIÓN EN EDUCACIÓN SUPERIOR: LA PERCEPCIÓN DE

LOS TUTORES DEL PROGRAMA PIANE-UC

Anastasiou, D., \& Kauffman, J. (2011). A social constructionist approach to disability: implications for special education. Exceptional Children, 77(3), 367-384.

Barton, L. (2009). Estudios sobre discapacidad y la búsqueda de la inclusividad: Observaciones. Revista de Educación, 349, 137-152.

Borland, J., \& James, S. (1999). The learning experience of students with disabilities in higher education. Disability \& Society, 14(1), 85-101.

Darrow, A. (2009). Barriers to effective inclusion and strategies to overcome them. General Music Today, 22(3), 29-31.

Da Silva, N., Guilhem, D., \& Dornelles, E. (2010). Modelo social: un nuevo abordaje para el tema deficiencia. Revista Latino-Americana de Enfermagem, 18(4), 816-823.

Dutta, A., Kundu, M., \& Schiro-Geist, C. (2009). Coordination of postsecondary transition services for students with disabilities. Journal of Rehabilitation, 75(1), 10-17.

Echeíta, G., \& Verdugo, M. (2004). La Declaración de Salamanca sobre Necesidades Educativas Especiales 10 años después. España: Publicaciones del INICO.

Espinoza, O., \& González, L. (2008). Perfil socioeconómico del estudiantado que accede a la Educación superior en Chile (1990-2003). Estudios Pedagógicos, 33(2), 45-57.

García, G. (2013). Red nacional de educación superior inclusiva en Chile: contexto y misión. En L. Pérez, A. Fernández y S. Katz, (Comps.), Discapacidad en Latinoamérica: Voces y experiencias universitarias (pp. 133-148). Buenos Aires: Editorial de la Universidad Nacional de La Plata.

Glaser, B., \& Strauss, A. (1967). The Discovery of grounded theory: Strategies for qualitative research. New York: Aldine de Gruyter.

Hernández, R., Fernández, C., \& Baptista, M. (2010). Metodología de la investigación. México: Mc Graw Hill.

Hockings, C., Brett, P., \& Terentjevs, M. (2012). Making a difference inclusive learning and teaching in higher education through open educational resources. Distance Education, 33(2), 237-252.

IESALC, UNESCO. (2005). Informe Final: Integración de las personas con discapacidad en la educación superior en Chile. Documento preparado por F. González y P. Araneda. Recuperado de http://www.fonadis.cl/

Jordán de Urries, B., \& Verdugo, M. (2014) Aproximación al problema de la inactividad en las personas con discapacidad. Revista del Ministerio de Empleo y Seguridad Social, 11(1), 63-76.

Katsiyannis, A., Zhang, D., Landmark, L., \& Reber, A. (2009). Postsecundary education for individuals with disabilities. Journal of Disability Policy Studies, 20(1), 35-45.

Kioko, V., \& Makoelle, T. (2014). Inclusion in Higher Education: Learning Experiences of Disabled Students at Winchester University. International Education Studies, 7(6), 106-118.

Konur, O. (2006). Teaching disabled students in higher education. Teaching in Higher Education, 11(3), 351-363.

Kowalsky, R., \& Fresko, B. (2002). Peer Tutoring for College Students with Disabilities. Higher Education Research \& Development, 21(3). doi.org/10.1080/0729436022000020760

Lissi, M., Zuzulich, S., Hojas, A., Achiardi, C., Salinas, M., \& Vásquez, A. (2013). En el camino hacia la educación superior inclusiva en Chile. Santiago de Chile: Pontificia Universidad Católica de Chile.

Lissi, M. R., Onetto, V., Zuzulich, M. S., Salinas, M., \& González, M. (2012). Tutorías de pares para favorecer la inclusión de estudiantes con discapacidad. Diálogos, 1(2), 26-29.

Lissi, M., Zuzulich, S., Salinas, M., Achiardi, C., Hojas A., \& Pedrals, N. (2009) Discapacidad en contextos universitarios: experiencia del PIANE UC en la Pontificia Universidad Católica de Chile. Calidad en la Educación, 30, 306-324.

Liasidou, A. (2014). Critical disability studies and socially just change in higher education. British Journal of Special Education, 41(2), 120-135.

Lou, M. (2011). Discapacidad: concepto y modelos explicativos. En M. Lou (Dir.), Atención a las necesidades educativas específicas (pp.18-38). Madrid: Pirámide. 
Medrano, D. (2009). Estudiantes universitarios con discapacidad: Análisis de los relatos de sus vivencias en el ámbito educativo (Tesis de Magíster). Pontificia Universidad Católica de Chile, Santiago de Chile.

Ministerio de Planificación, Chile. (2010). Ley 20.422. Recuperado de http://www.senadis.gob.cl/ pag/177/557/ley_n20422.

Moreno, M. (2006). Integración/inclusión de las personas con discapacidad en la Educación Superior. En Instituto Internacional de la UNESCO para la Educación Superior en América Latina y el Caribe. Informe sobre la educación superior en América Latina y el Caribe. 2000- 2005: La metamorfosis de la educación superior. Caracas: Editorial Metrópolis.

Muccio, L. (2012) Head start instructional professionals' perceptions and practices: facilitators and barriers for including young children with disabilities (Tesis de doctorado). George Mason University, Washington DC, Estados Unidos.

Naciones Unidas. (2006). Convención sobre los Derechos de las Personas con Discapacidad. Ginebra: Autor

Padilla, A. (2011). Inclusión educativa de personas con discapacidad. Revista Colombiana de Psiquiatría, 40(4), 670-699.

Palacios, A. (2008). El modelo social de discapacidad: Orígenes, caracterización y plasmación en la Convención Internacional sobre los Derechos de las personas con discapacidad. España: Ediciones Cinca.

Pivik, J., McComas, J., \& Laflamme, M. (2002). Barriers and facilitators to inclusive education as reported by students with physical disabilities and their parents. Exceptional Children, 61(1), 97-107.

Sachs, D., \& Schreuer, N. (2011). Inclusion of students with disabilities in higher education: Performance and participation in student's experiences. Disability Studies Quarterly, 31(2), 1593-1561.

Sánchez, A. (2010). Identificación de barreras educativas en el proceso de transición de la educación media a la educación superior en personas sordas: un estudio exploratorio (Tesis de Maestría). Universidad Nacional de Colombia, Bogotá, Colombia. Recuperado de http://www.bdigital. unal.edu.co/8718/

Sandín, M. (2003). Investigación Cualitativa en Educación: Fundamentos y tradiciones. Madrid: McGrawHill.

Strauss, A., \& Corbin, J. (2002). Bases de la investigación cualitativa: Técnicas y procedimientos para desarrollar la teoría fundamentada. Newbury Park, CA: Sage.

Vogel, G., Fresko, B., \& Wertheim C. (2007). Peer tutoring for college students with learning disabilities: perceptions of tutors and tutees. Journal of Learning Disabilities, 40(43), 485-493. 
